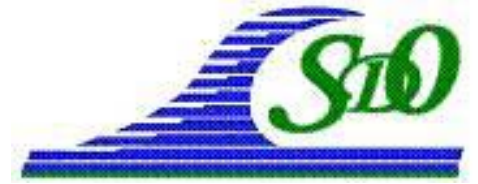

XI ${ }^{\text {èmes }}$ Journées Nationales Génie Côtier - Génie Civil

Les Sables d'Olonne, 22-25 juin 2010

DOI:10.5150/jngcgc.2010.084-S

(c) Editions Paralia CFL

disponible en ligne - http://www.paralia.fr - available online

\title{
Appontements flottants de nouvelle génération pour l'accueil des frégates multi-missions (FREMM)
}

\author{
Yves STASSEN $^{1}$, François LESNE $^{2}$, Yves ROBIN $^{3}$, Simon HOFLACK $^{4}$
}

1. CHARIER SA, 15 boulevard Marcel Paul, 44800 Saint Herblain, France. ystassen@charier.fr

2. INGEROP, 2 rue de la Rigourdière, CS 41729, 35517 Cesson Sévigné cedex, France. françois.lesne@ingerop.com

3. DCNS, CS 72837 F, 29228 Brest cedex 2, France.

yves.robin@dcnsgroup.com

4. PRINCIPIA, 1 rue de la Noë, BP22110, 44321 Nantes cedex 3, France.

Simon.hoflack@principia.fr

\section{Résumé :}

La Direction Régionale du Service d'Infrastructure de la Défense (DRSID) de Brest a lancé en février 2009 un appel d'offre pour la conception, la réalisation, l'entretien et la maintenance d'appontements dédiés à l'accueil des futures frégates européennes multimissions FREMM. Le cahier des charges fonctionnel laissait une large initiative dans la conception de l'ouvrage destiné aux fonctions suivantes : accostage et amarrage des navires, accès des piétons et véhicules, raccordement aux réseaux et servitudes du navire. Le caractère innovant du projet lauréat réside dans la conception d'un ouvrage flottant à double pont permettant une répartition sécurisée, ergonomique et évolutive de ces fonctions sur deux niveaux. L'ouvrage se distingue également par son système d'ancrage constitué de chaînes semi-tendues, ancrées à une extrémité sur le quai existant et à l'autre extrémité sur un ouvrage gravitaire permettant de valoriser les sédiments de dragage sous forme de lest.

Mots-clés : Appontement - Flottant - FREMM - Accostage - Amarrage

\begin{abstract}
:
French Department of Defence (DRSID, Brest) has launched in February 2009 an invitation to tender for the design, build and maintenance of piers dedicated to the new generation of European frigate (FREMM). The functional specifications were open to a wide range of technical solutions to satisfy the following requirements: ship berthing and mooring, pedestrian and vehicle access, ship connection to fluids and energies. The awarded project is based on the innovative concept of double deck floating pier which allows for secured, ergonomic and evolutive repartition of these activities. The mooring system of the pier consists of taut chains anchored, at one side, on the existing quay and, at the other side, on a gravity based structure ballasted with dredged sediment.
\end{abstract}

Keywords: Pier - Floating - FREMM - Berthing - Mooring 
Thème 5 - Ouvrages portuaires, côtiers et offshore

\section{Introduction}

La Direction Régionale du Service d'Infrastructure de la Défense (DRSID) de Brest a lancé en février 2009 un appel d'offre pour la conception, la réalisation, l'entretien et la maintenance de deux lignes d'accostage et de stationnement dédiées à l'accueil des futures frégates européennes multi-missions FREMM. Ces appontements sont destinés à remplacer les lignes existantes au quai des flottilles, dans le port militaire de Brest.
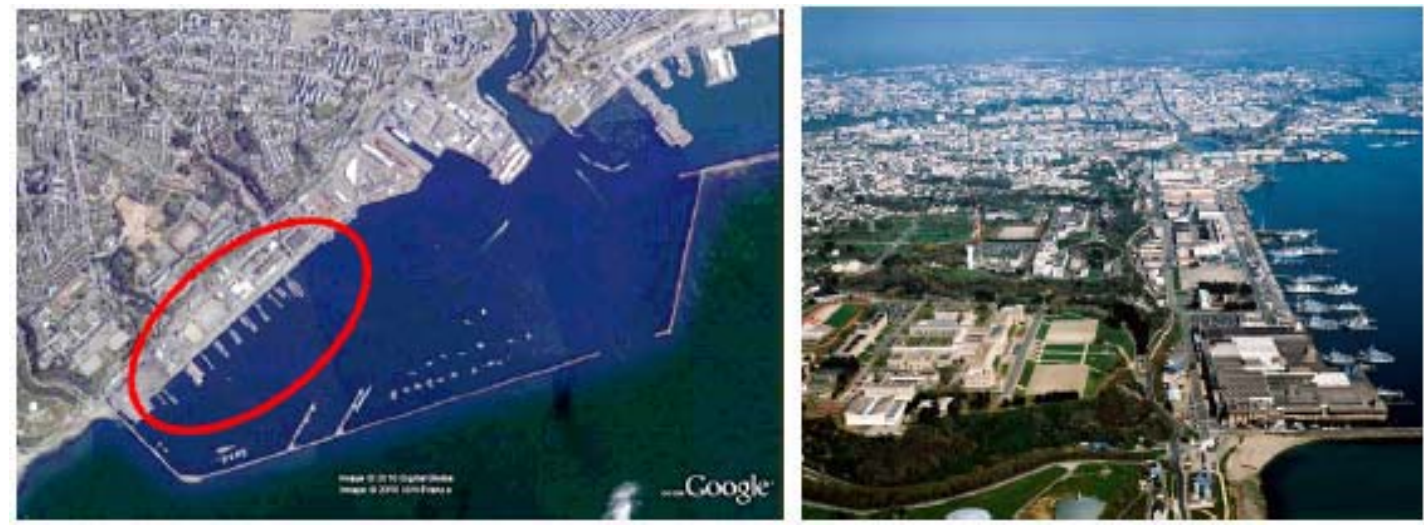

Figure 1. Situation du projet : rade de Brest, quai des flottilles.

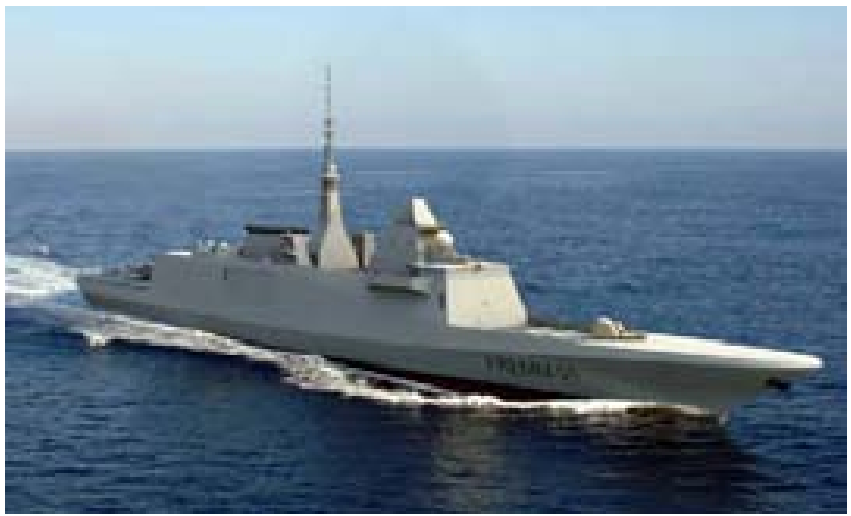

Figure 2. Nouvelle frégate européenne mulit-mission FREMM.

Le cahier des charges fonctionnel établi par la Marine laissait aux entreprises candidates une large initiative dans la conception d'un ouvrage prototype destiné aux principales fonctions suivantes : accostage et amarrage des navires, embarquement et débarquement des piétons, accessibilité aux véhicules lourds et légers, raccordement aux réseaux et servitudes du navire (hydrocarbure et électricité haute tension en particulier).

Le groupement d'entreprises lauréat, Charier (mandataire), Ingerop (ingénierie), Semen tp (génie civil, dragage), Demathieu \& Bard (génie civil), Ducrocq Ingénierie et Process (construction métallique), DCNS (réseaux, maintenance), a proposé un projet innovant sur le principe d'un ouvrage flottant à double pont. Le groupement est assisté de PRINCIPIA pour la réalisation des études hydrodynamiques. 


\section{XI ìmes Journées Nationales Génie Côtier-Génie Civil}

Les Sables d'Olonne, 22-25 juin 2010

\section{Description fonctionnelle de l'ouvrage}

\subsection{Principales exigences}

Les lignes d'accostage actuelles sont constituées d'appontements métalliques ancrés sur chaînes et accessibles par une passerelle piéton depuis le quai des flottilles. Elles ont pour fonctions principales: (1) l'accostage et l'amarrage des navires, (2) l'embarquement et le débarquement de piétons, (3) l'avitaillement des navires (vivres, fluides, énergies,...), (4) le raccordement aux réseaux de courants faibles, (5) le stockage temporaire avant embarquement ou débarquement.

Ces appontements permettent d'accueillir les frégates actuellement en service, d'une longueur de $120 \mathrm{~m}$ pour un déplacement de $3800 \mathrm{~T}$. Les nouvelles frégates multimissions (FREMM) de la Marine nationale, appelées au service actif pour l'année 2012, atteignent une longueur de $142 \mathrm{~m}$ pour un déplacement de $6200 \mathrm{~T}$. Ces évolutions majeures de la flotte justifient le remplacement et l'évolution des appontements.

Outre les fonctions dévolues aux lignes actuelles, le programme de l'opération exprime une attente particulière sur: (1) l'accessibilité de véhicules lourds et légers, (2) l'accès aux réseaux et servitudes (distribution hydrocarbure, électricité basse et haute tension $6.6 \mathrm{kV} 60 \mathrm{~Hz}$, réseaux d'eau et d'air comprimé, évacuation des résidus de fond de cale), (3) l'ergonomie générale de l'ouvrage et plus particulièrement de son interface avec le navire.
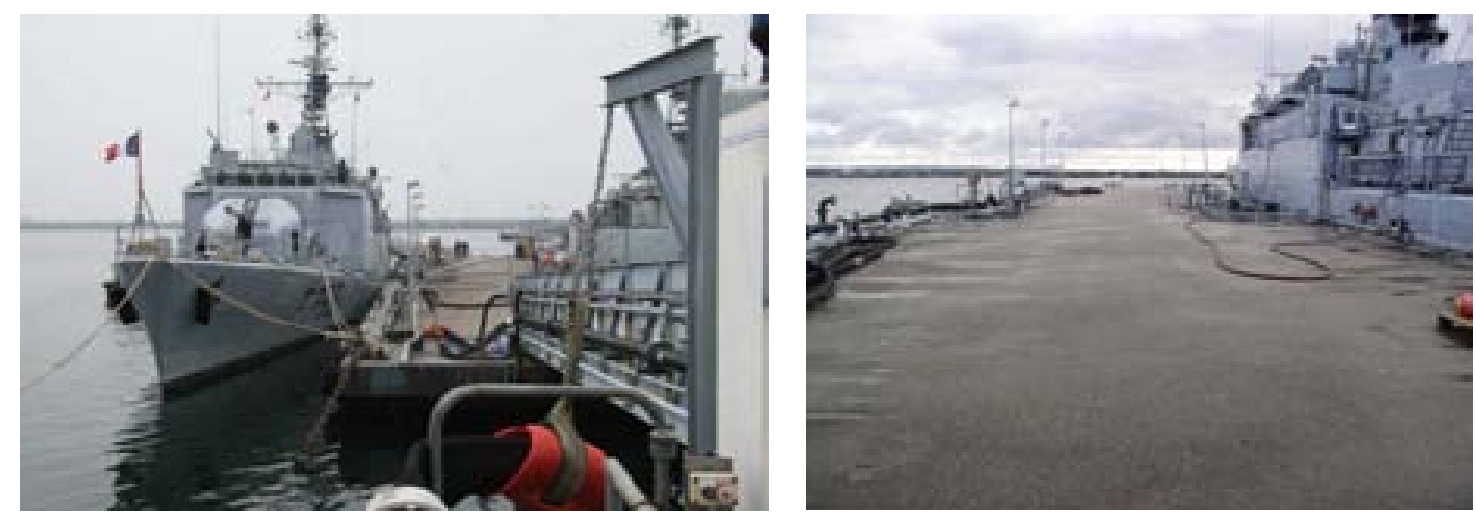

Figure 3. Lignes d'accostage actuelles.

\subsection{Conception générale}

L'analyse du cahier des charges a conduit le groupement à proposer une conception innovante basée sur le principe d'un appontement à double niveau de pont. Cette conception est guidée par les objectifs suivants :

- séparation, rationalisation des flux (personnel, véhicules, réseaux) ;

- accessibilité permanente depuis le quai des flottilles, quel que soit le niveau de marée ;

- ergonomie des interfaces appontement/navire. 
L'ouvrage est ainsi constitué :

- d'un caisson flottant monolithique en béton armé, de $160 \mathrm{~m}$ de long par $17 \mathrm{~m}$ de large, avec un tirant d'eau de $3.40 \mathrm{~m}$ et un franc-bord de $1.50 \mathrm{~m}$;

- d'une superstructure également en béton armé, de type dalle sur poutres et poteaux, de $14 \mathrm{~m}$ de large et $2.80 \mathrm{~m}$ de haut.

L'appontement est implanté à 30m du quai des flottilles, perpendiculairement à celui-ci. Il est amarré sur chaînes semi-tendues : 1) côté nord sur le quai existant, moyennant la création de massifs d'ancrage spécifiques, 2) côté sud sur un ouvrage gravitaire circulaire, baptisé "caisson musoir", qui permet de valoriser les sédiments de dragage sous forme de lest. Le pont supérieur est accessible aux piétons et véhicules par une passerelle métallique supportant également le passage des réseaux. Le pont inférieur est accessible aux piétons par escaliers depuis le pont supérieur.

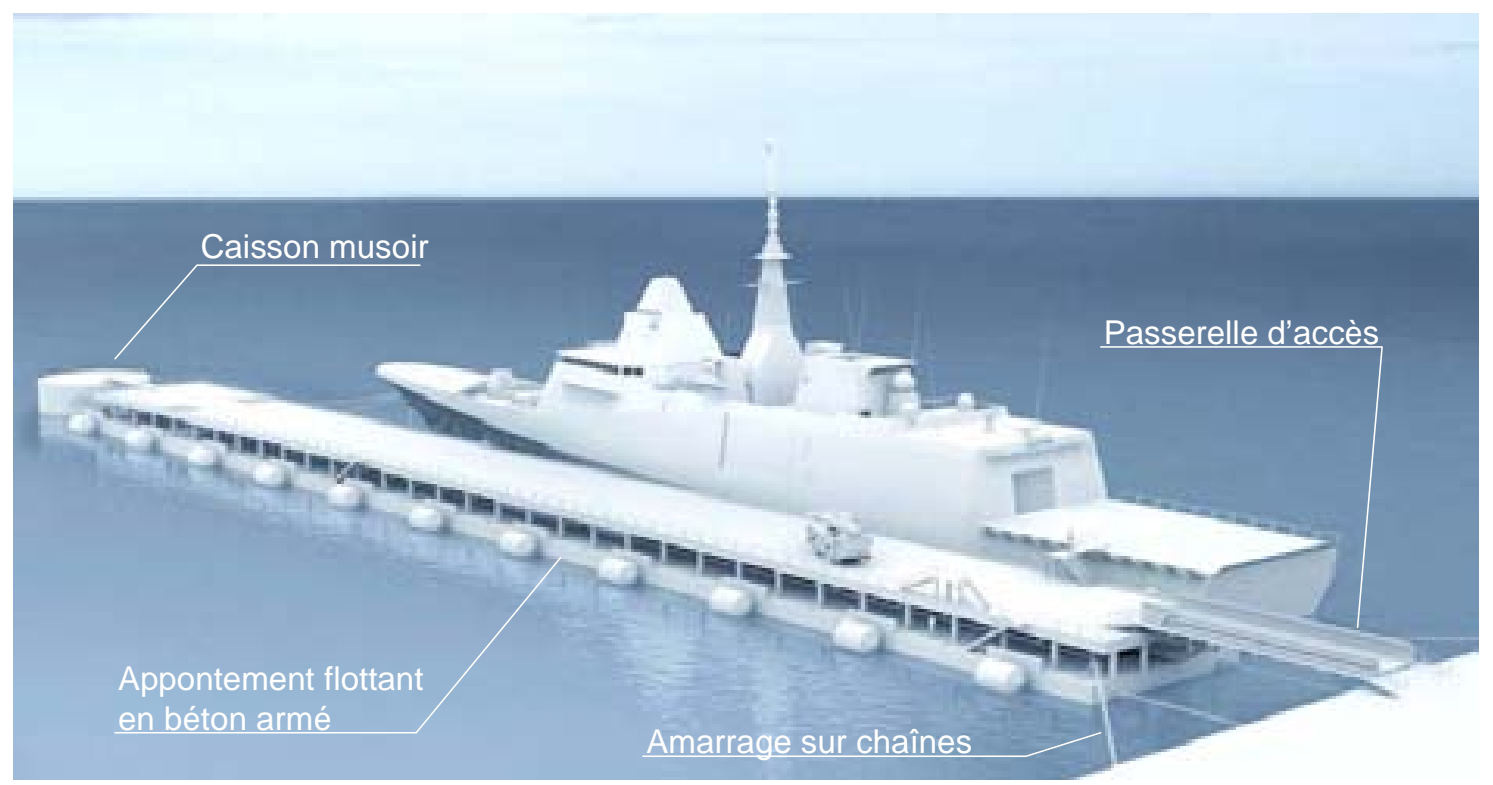

Figure 4. Vue d'ensemble du projet.

\subsection{Principe de séparation des flux}

Durant son exploitation l'ouvrage doit permettre de faire cohabiter simultanément et en toute sécurité une circulation piétonne (accès des équipages à bord des navires), une circulation de véhicules lourds (grues mobiles et camions d'avitaillement) ainsi que des réseaux techniques sensibles (alimentation gasoil, électricité haute tension). La séparation de ces flux sur deux ponts superposés indépendants offre un niveau de sécurité et d'ergonomie élevé à l'ensemble des usagers.

Le pont supérieur est accessible aux piétons et véhicules. Il est entièrement dégagé de toute servitude technique. Il est destiné à l'avitaillement des navires par camions et grues mobiles, à l'accès à bord des équipages et au stockage temporaire de colis avant ou après débarquement. 

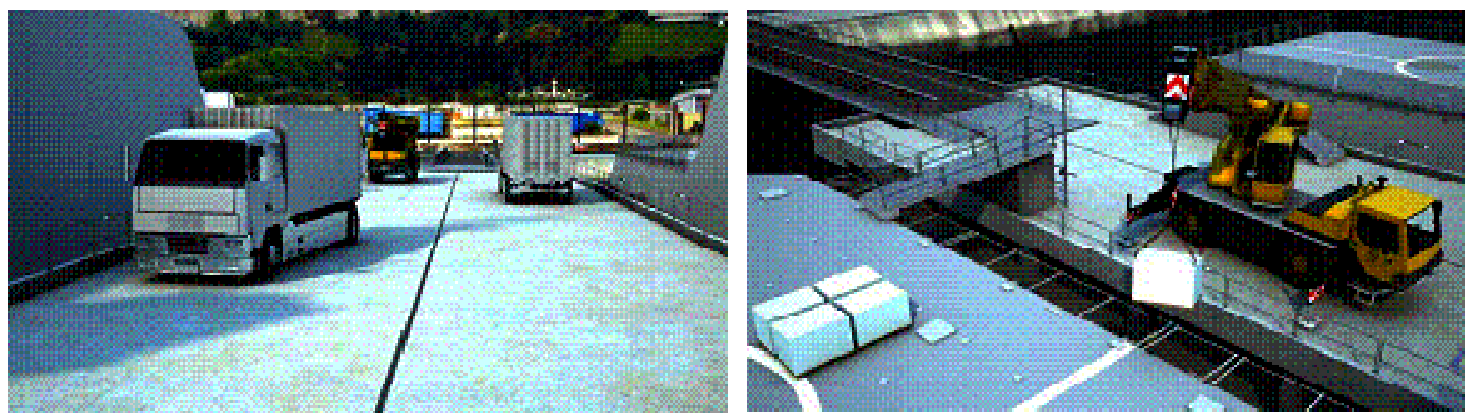

Figure 5. Vue du pont supérieur de l'appontement.

Le pont inférieur est destiné au personnel d'exploitation. Il supporte l'ensemble des équipements nécessaires aux servitudes du navire (lamanage, alimentation en fluides et énergies, local de transformation électrique). Il se décompose en 5 travées délimitées par les files de poteaux de la structure : une travée centrale dédiée à l'implantation de l'ensemble des réseaux, deux coursives latérales offrant un espace de travail vaste et dégagé pour les opérations de raccordement des réseaux aux navires, deux coursives de lamanage à ciel ouvert réservées aux manœuvres d'accostage et d'amarrage.

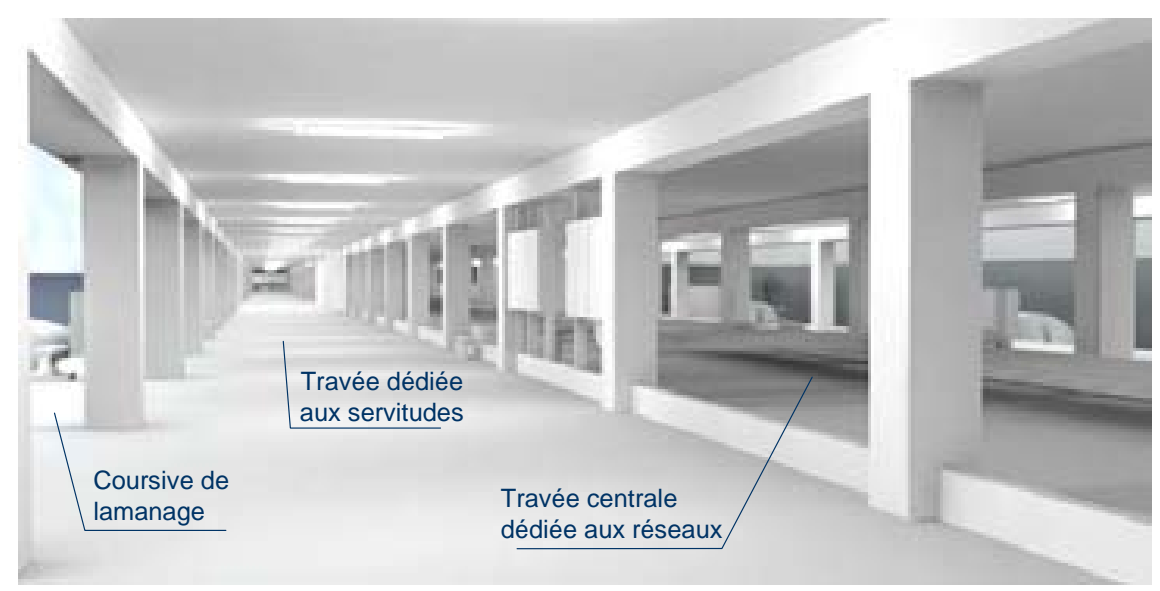

Figure 6. Vue du pont inférieur de l'appontement.

\subsection{Accessibilité permanente}

Le cahier des charges exige l'accessibilité permanente de l'ouvrage aux véhicules, avec une pente maximale de $13 \%$ sur la passerelle d'accès, quel que soit le niveau de marée. Les contraintes altimétriques pour la conception de l'ouvrage d'accès sont les suivantes :

- niveau du quai des flottilles

$+8.50 \mathrm{~m} \mathrm{CM}$

- niveau de plus basse mer $0.00 \mathrm{~m} \mathrm{CM}$;

- niveau de plus haute mer $+8.40 \mathrm{~m} \mathrm{CM}$. 
Le pont supérieur de l'appontement s'élève à $4.30 \mathrm{~m}$ au dessus du niveau d'eau $(1.50 \mathrm{~m}$ de franc-bord $+2.80 \mathrm{~m}$ de hauteur de superstructure). Il se positionne ainsi à un niveau moyen (mi-marée à $+4.20 \mathrm{~m} \mathrm{CM}$ ) identique à celui du quai des flottilles (+8.50 m CM). Cette disposition limite à $\pm 4.20 \mathrm{~m}$, aux plus fortes marées, la dénivelée à franchir pour l'accès à l'appontement.

La pente admissible de $13 \%$ est alors respectée avec une passerelle de courte longueur (35 m) qui présente un double avantage: (1) emprise limitée sur l'appontement, libération de la zone d'accès au pont arrière des FREMM, (2) conception structurelle standard, sans appui intermédiaire.

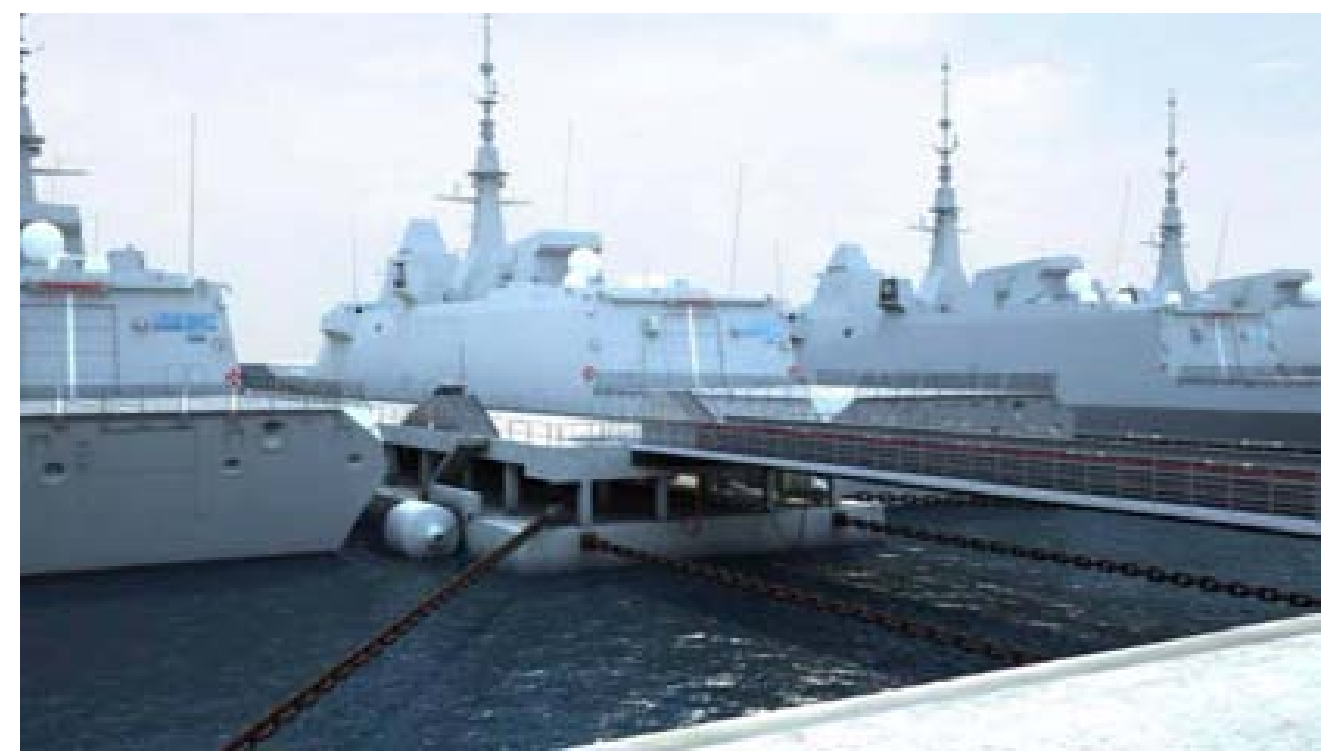

Figure 7. Passerelle d'accès, situation mi-marée (passerelle horizontale).

\subsection{Ergonomie de l'interface navire / appontement}

Du point de vue de la Marine nationale, utilisateur final de l'infrastructure, les interfaces entre l'appontement et les navires constituent l'enjeu majeur du projet. Ces interfaces se situent en particulier au niveau des moyens d'accès à bord des équipages, des dispositifs d'accostage et d'amarrage, ainsi que des points de raccordement des réseaux fluides et énergies.

L'accès des équipages à bord des navires est facilité par le niveau élevé du pont supérieur au-dessus du plan d'eau, proche de celui du pont arrière des FREMM, qui permet l'utilisation de coupées de faible dénivelée et de faible encombrement.

Sur le pont inférieur, les coursives de lamanage sont conçues à ciel ouvert, en débord de $1.50 \mathrm{~m}$ par rapport au pont supérieur. Cette disposition offre aux lamaneurs la visibilité nécessaire sur le navire et le milieu environnant lors des opérations d'accostage et d'amarrage. Elle garantit un espace suffisant entre l'étrave des navires et la structure de l'appontement dans le cas d'un accostage accidentel en incidence oblique. 


\section{XI ìmes Journées Nationales Génie Côtier-Génie Civil}

Les Sables d'Olonne, 22-25 juin 2010

Les points de raccordement aux différents réseaux de servitude sont quant à eux éloignés du front d'accostage et disposés à l'abri des intempéries sous la dalle du pont supérieur.

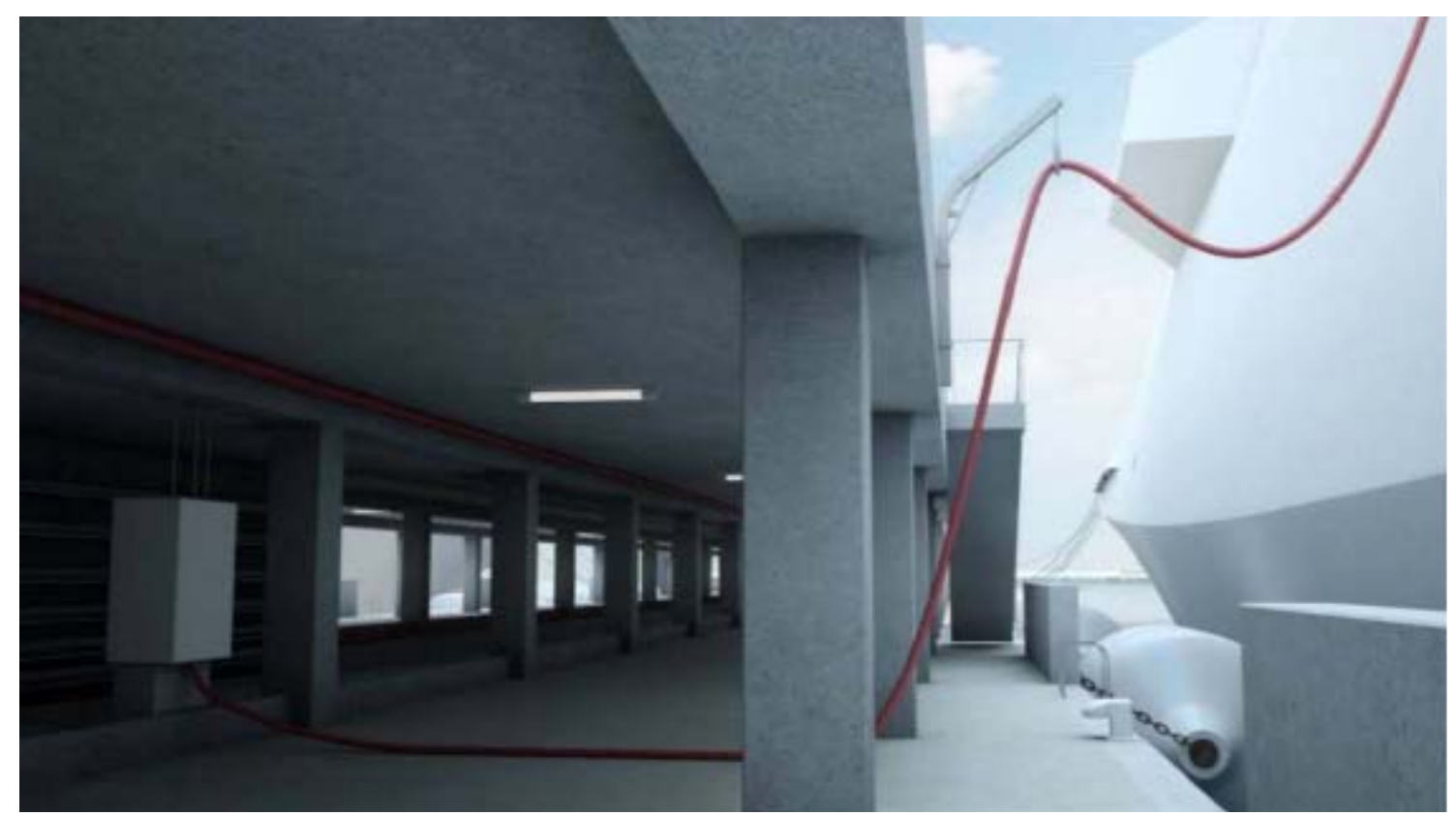

Figure 8. Pont inférieur : coursive de lamanage et point de raccordement électrique.

\section{Comportement à l'accostage et à l'amarrage}

\subsection{Système d'ancrage de l'appontement}

L'appontement est ancré au moyen de 4 chaînes à chacune de ses extrémités : 2 chaînes obliques et 2 chaînes longitudinales.

L'ancrage est réalisé côté nord sur le quai existant et côté sud sur le caisson musoir.

Les lignes obliques sont dimensionnées pour reprendre l'ensemble des efforts d'ancrage de l'appontement, y compris les efforts générés en situation d'accostage accidentel. Les lignes longitudinales offrent une sécurité supplémentaire en cas de dépassement significatif de la vitesse d'accostage accidentel.

Chaque chaînes obliques est montée sur appareil amortisseur implanté sur l'appontement et permettant d'écrêter les surtensions générées lors des accostages.

Les calculs de pré-dimensionnements menés au stade de l'avant-projet conduisent à retenir de chaînes à étai de diamètre DN84 (charge d'épreuve $3610 \mathrm{kN}$, charge de rupture $5160 \mathrm{kN}$ ).

\subsection{Défenses d'accostage}

Le front d'accostage est équipé de défenses flottantes en mousse à faible module de réaction (mousse polyéthylène avec enveloppe polyuréthane). Ces défenses sont 
Thème 5 - Ouvrages portuaires, côtiers et offshore

adaptées à l'accostage de navires militaires : elles génèrent des pressions sur coque de faible intensité et s'adaptent bien aux différentes formes de carène de la flotte militaire. Sur chaque bord, l'appontement est équipé de 11 défenses réparties tous les 15 mètres. Les défenses retenues ont une longueur de $4.00 \mathrm{~m}$ et un diamètre de $2.50 \mathrm{~m}$. Ces défenses ont une capacité d'absorption d'énergie nominale comprise entre $500 \mathrm{kN}$ m et $800 \mathrm{kN}$ m selon la densité de mousse retenue. Les défenses les plus souples permettent de limiter les pressions sur coque à $10 \mathrm{~T} / \mathrm{m}^{2}$ sur l'ensemble de leur plage de fonctionnement. Cette valeur est conforme aux pressions admissibles spécifiées sur la FREMM.
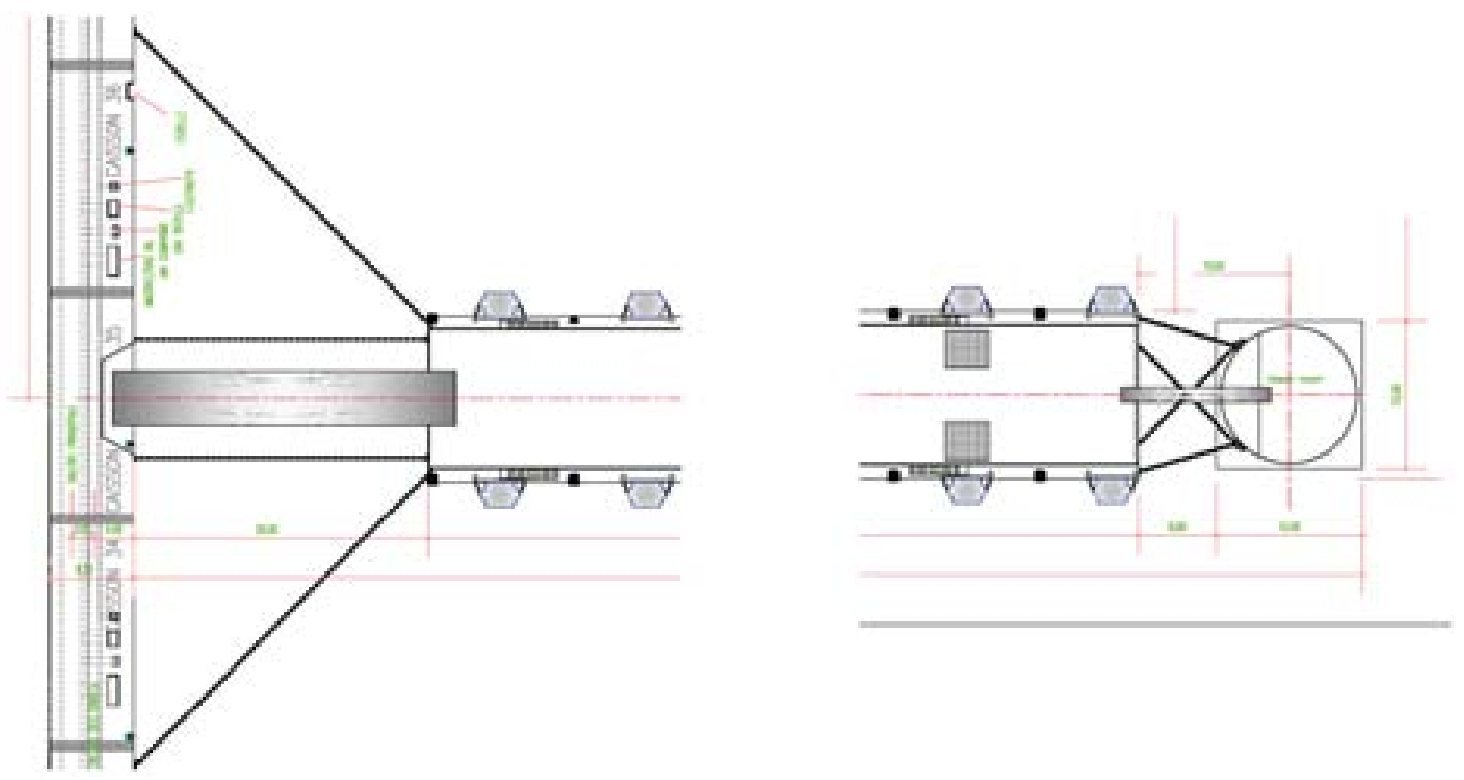

Figure 9. Ancrage de l'appontement (côté quai et côté musoir).

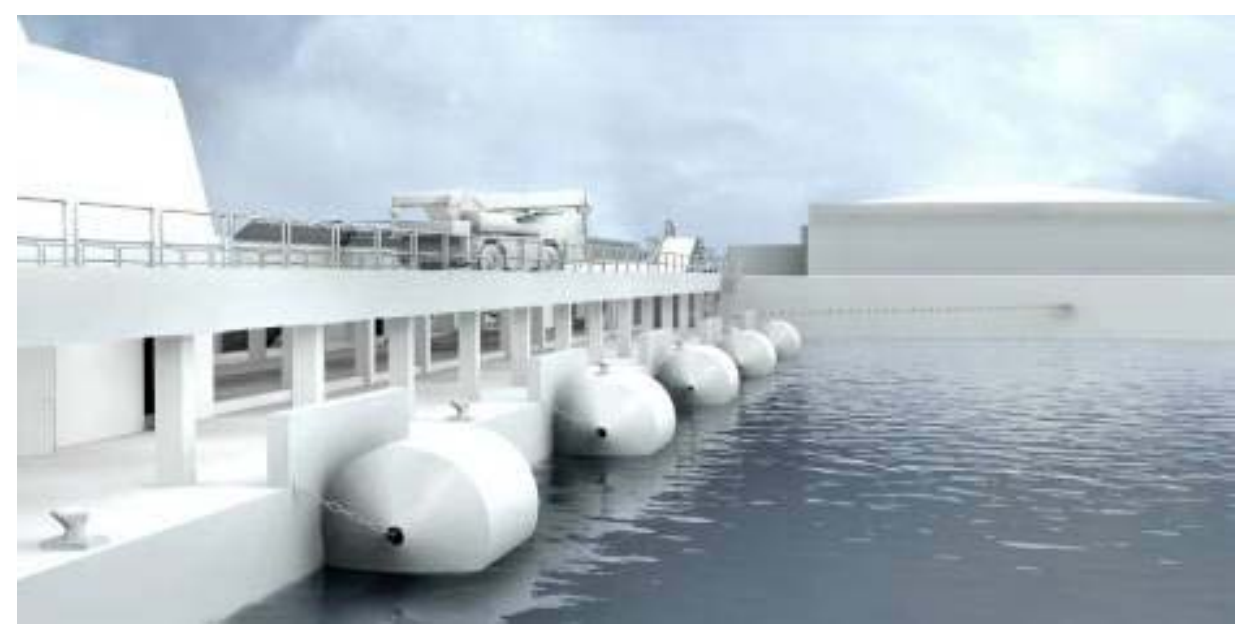

Figure 10. Front d'accostage équipé de défenses flottantes. 


\subsection{Etude d'amarrage}

Dans le cadre des études d'avant-projet, l'étude du comportement de l'appontement à l'amarrage a été menée en présence d'une FREMM sur chaque bord, au moyen du logiciel hydrodynamique DIODORE ${ }^{\mathrm{TM}}$.

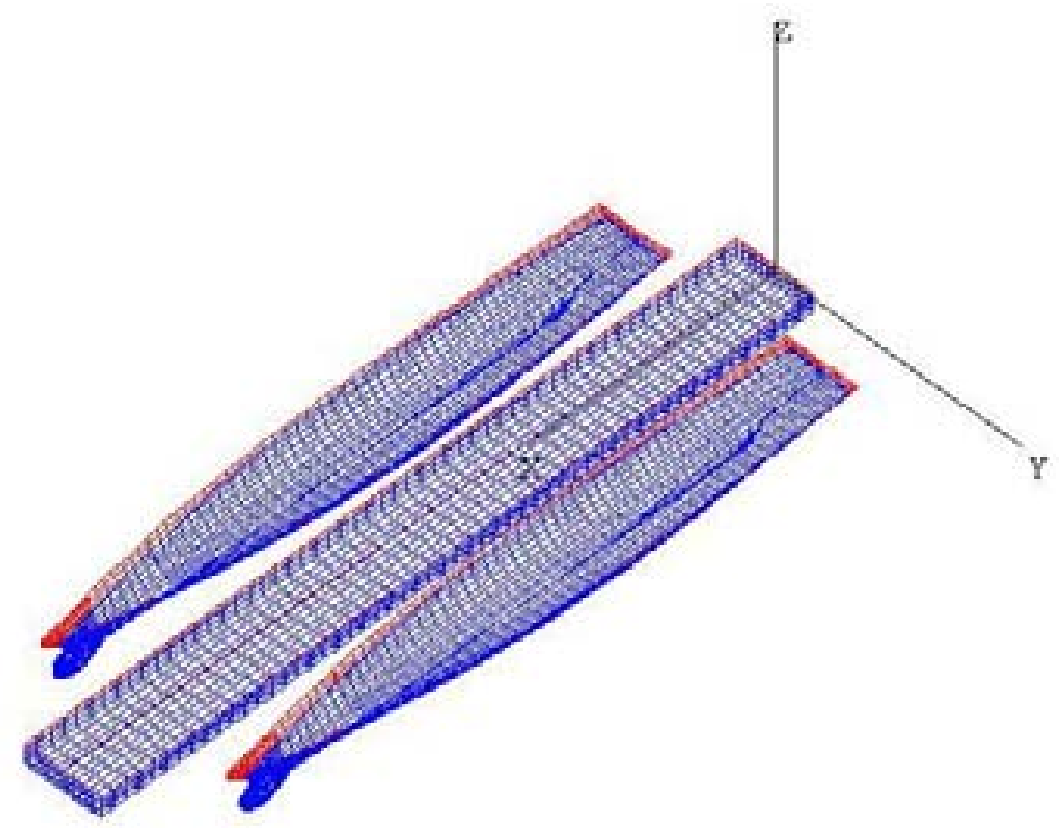

Figure 11. Maillage du modèle DIODORE ${ }^{T M}$.

Les conditions climatiques extrêmes suivantes ont été considérées (situation ELU) :

- vent moyen de $37 \mathrm{~m} / \mathrm{s}$ (moyenne sur 10 minutes), perpendiculaire à l'appontement (vent irrégulier modélisé par un spectre de Davenport) ;

- houle irrégulière de hauteur significative $\mathrm{H}_{\mathrm{s}}=0.66 \mathrm{~m}$ et de période de pic $\mathrm{T}_{\mathrm{p}}=2.48 \mathrm{~s}$, perpendiculaire à l'appontement (spectre de Jonswap, $\gamma=3.3$ ) ;

- courant constant de 1 nœud, perpendiculaire à l'appontement.

\subsection{Etude d'accostage}

L'étude du comportement de l'ouvrage à l'accostage est menée au moyen du logiciel LS-DYNA (résolution des équations de la dynamique de corps déformables) couplé à DIODORE $^{\mathrm{TM}}$ pour le calcul des efforts hydrodynamiques. Les défenses d'accostage sont modélisées par des plaques sur ressorts, de dimensions et de raideur équivalentes aux défenses d'accostage prévues. 


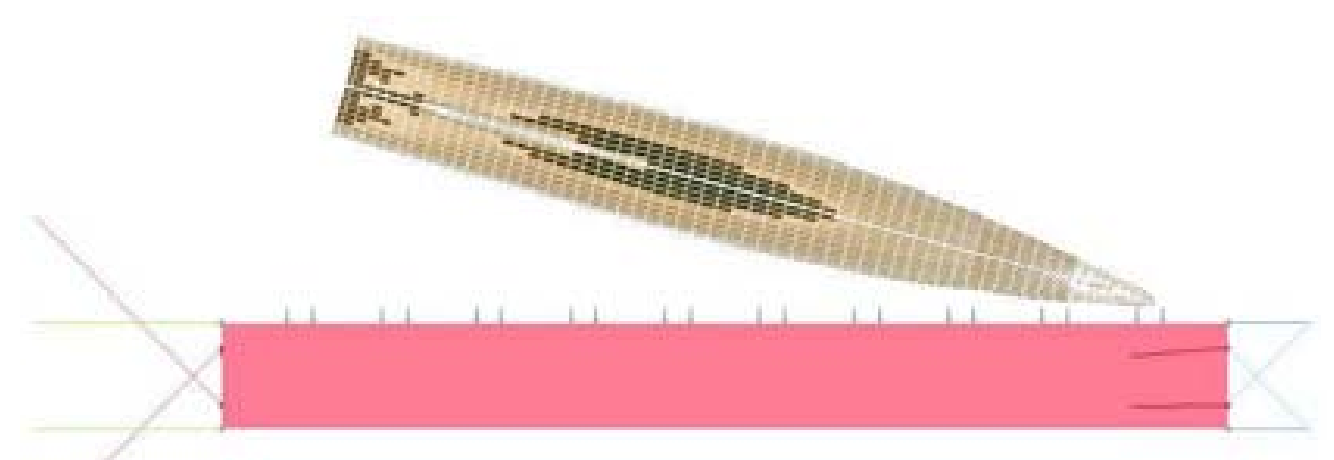

Figure 12. Modélisation LS-DYNA, accostage FREMM en incidence oblique $\left(15^{\circ}\right)$.

Dans le cadre des études d'avant-projet, les accostages ont été étudiés sous incidence nulle et sous une incidence oblique de $15^{\circ}$, avec des vitesses de 0.5 nœud en situation normale et 1.0 nœud en situation accidentelle. Ces simulations sont réalisées avec un pas de temps très court $\left(9.10^{-5} \mathrm{~s}\right.$, déterminé automatiquement par LS-DYNA) permettant de suivre précisément l'évolution des efforts lors de l'impact.

\subsection{Principaux résultats}

Les tensions maximums obtenues dans les chaînes d'ancrage de l'appontement sont reportées dans le tableau ci-dessous.

Tableau 1. Tensions maximum dans les ancrages de l'appontement.

\begin{tabular}{ll}
\hline Situation & $\begin{array}{l}\text { Tension maximum dans les } \\
\text { chaînes d'ancrage }\end{array}$ \\
\hline Amarrage ELU & $2500 \mathrm{kN}$ \\
Accostage $0.5 \mathrm{NM}$ - incidence $0 \mathrm{deg}$ & $1190 \mathrm{kN}$ \\
Accostage $1.0 \mathrm{NM}$ - incidence $0 \mathrm{deg}$ & $2320 \mathrm{kN}$ \\
Accostage $0.5 \mathrm{NM}$ - incidence $15 \mathrm{deg}$ & $780 \mathrm{kN}$ \\
Accostage $1.0 \mathrm{NM}$ - incidence $15 \mathrm{deg}$ & $1550 \mathrm{kN}$ \\
\hline
\end{tabular}

La figure 13 présente l'historique des tensions dans les lignes lors de l'accostage à 1.0 nœud sous incidence nulle. Ce graphique fait apparaître :

- un mouvement d'embardée de l'appontement, conduisant à une mise en tension successive des lignes bâbord (1 et 3) puis tribord (ligne 4), avec un déphasage correspondant à la période propre en embardée du système (environ $9 \mathrm{~s}$ ) ;

- un mouvement de lacet de l'appontement, conduisant à une mise en tension successive des lignes nord (ligne 1) puis sud (ligne 3), avec un déphasage correspondant à la période propre en lacet du système (environ $5 \mathrm{~s}$ ). 


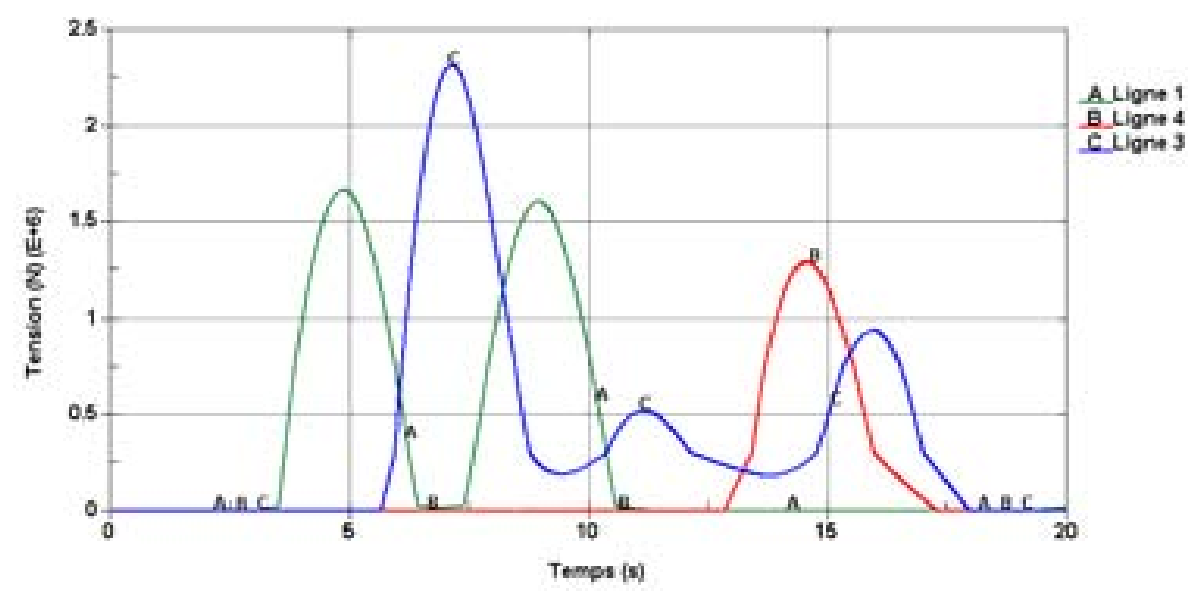

Figure 13. Historique des tensions dans les chaînes (accostage $1.0 \mathrm{NM}$, incidence $0^{\circ}$ ).

Les enveloppes de mouvements obtenues sont reportées dans le tableau 2.

Tableau 2. Enveloppes maximums des mouvements de l'appontement.

\begin{tabular}{ll}
\hline $\begin{array}{l}\text { Dérive } \\
\text { (houle, vent, courant) }\end{array}$ & $60 \mathrm{~cm}$ \\
\hline $\begin{array}{l}\text { Mouvement sur houle } \\
\text { Embardée }\end{array}$ & $\pm 30 \mathrm{~cm}$ \\
\hline $\begin{array}{l}\text { Mouvement sur houle } \\
\text { Pilonnement }\end{array}$ & $\pm 2 \mathrm{~cm}$ \\
\hline $\begin{array}{l}\text { Vitesses } \\
\text { (toutes directions confondues, en tout point de l'appontement) }\end{array}$ & $0.16 \mathrm{~m} / \mathrm{s}$ \\
\hline $\begin{array}{l}\text { Accélérations } \\
\text { (toutes directions confondues, en tout point de l'appontement) }\end{array}$ & $0.14 \mathrm{~m} / \mathrm{s}^{2}$ \\
\hline
\end{tabular}

Les déplacements, vitesses, accélérations de l'appontement sont faibles et parfaitement compatibles avec les contraintes d'exploitation de l'ouvrage : accès à bord, circulation de piétons et véhicules, implantation et utilisation d'équipements techniques, confort à bord.

\section{Conclusion}

L'appel d'offre lancé par la Direction Régionale du Service d'Infrastructure de la Défense de Brest a laissé aux entreprises candidates une large initiative dans la conception d'appontements prototypes destinés à l'accueil des frégates de nouvelle génération de la Marine nationale (FREMM).

Le projet retenu est basé sur le principe innovant d'un appontement flottant à double niveau de pont, garantissant une répartition sécurisée, ergonomique et évolutive des différentes fonctionnalités demandées au cahier des charges. 
Thème 5 - Ouvrages portuaires, côtiers et offshore

Les études hydrodynamiques menées en phase d'avant-projet au moyen des logiciels DIODORE $^{\mathrm{TM}}$ et LS-DYNA ont permis de valider le comportement de l'ouvrage en situation d'amarrage et d'accostage : tensions dans les lignes d'ancrage et mouvements de l'appontement.

Les études d'avant-projet détaillé se poursuivent en 2010, parallèlement à l'instruction du dossier d'autorisation administratif. Le démarrage des travaux est prévu mi-2011, pour une mise en service du premier appontement mi-2012. 